\title{
OLYMPUS
}

Your Vision, Our Future

\section{High-resolution, rapid image acquisition for studying biological structures and dynamic cellular processes}

\author{
The Olympus cell $\wedge$ R and cell $\wedge M$ imaging workstations are versatile and modular platforms, which allow \\ rapid multidimensional analysis of fluorescently labeled biological samples. In addition to wide-field \\ imaging, the systems can incorporate a white-light spinning disk confocal unit or additional lasers for \\ total internal reflection fluorescence microscopy (TIRFM).
}

Fluorescence microscopy is a key technique for cell biology, providing the capacity to monitor cellular activities in living tissue with submicrometer resolution in real time. In parallel with the continuing expansion of specific fluorescent probes, there have been quantum leaps in the capacity of hardware for imaging fluorescently labeled cells. Furthermore, relatively sophisticated modes of fluorescence imaging, such as TIRFM, are now available as off-the-shelf turn-key solutions. As a result, cell biologists are demanding imaging systems that are increasingly multifunctional, have greater temporal and spatial sensitivity, and can support the specific requirements of different users.

Olympus has developed the cell $\wedge R$ and cell $\wedge M$ to meet these expanding demands. These systems can be fully integrated with their IX (inverted) and BX (upright) microscopes. The combination of a motorized microscope with the cell $\wedge \mathrm{R}$ or cell $\wedge \mathrm{M}$ system allows the user to design complex multiparameter experimental protocols. For example multicolor, z-stack images can be acquired at any number of locations using variable time-lapse intervals, and can include brightfield images (Köhler, DIC or phase contrast) with online analysis. The advanced autofocus gives consistently clear images, and TTL pulse triggering provides precise high-speed control of external devices. Highly stable illumination is provided by the unique all-in-one MT10 and MT20 systems, which incorporate 150-watt xenon or mercury-xenon arc lamps, an 8-position excitation filter wheel and a 14-position attenuation shutter (7 positions for the MT10). The MT20 is capable of switching excitation wavelengths and attenuation filters in $58 \mathrm{~ms}$ (85 ms for the MT10). The excitation light is controlled by rapid shutters ( $1 \mathrm{~ms}$ in the MT20 and $5 \mathrm{~ms}$ for the MT10) to reduce unwanted overillumination or photobleaching. The cell $\wedge \mathrm{R}$ uses a control board running the Linux operating system independently of the imaging computer, ensuring the highest possible timing accuracy for every image. These systems

\section{Alan Kidger}

Olympus UK Ltd., Great Western Industrial Park, Dean Street, Southall, Middlesex UB2 4SB, UK. Correspondence should be addressed to A.K. (microscopy@olympus.uk.com).

PUBLISHED ONLINE 21 JUNE 2006; DOI:10.1038/NMETH893 support several charge-coupled device (CCD), as well as electron multiplying CCD cameras.

\section{Designing protocols, data acquisition and analysis}

The 'Experiment Manager' provides an intuitive drag-and-drop interface making it easy to devise even the most complex protocols. Icons relating to specific functions are simply selected and linked together in the order required. These command icons provide functions such as time-lapse, z-stack, autofocus and image acquisition. All experimental protocols are saved along with the corresponding data, allowing previously stored protocols to be reused. Data is stored in a user-specific archive with successive experiments being numbered sequentially. Multidimensional data sets (including $X Y Z$, time, color and stage position) are stored in the database, along with results of the real-time analysis and report files.

Image capture is coupled with peerless analysis software to provide great flexibility. Features range from basic measurements such as number, length, area and gray value, to advanced methods that include colocalization, spectral unmixing for optimized color
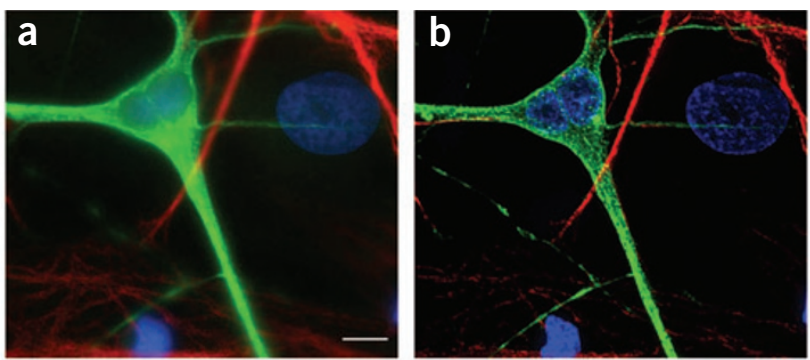

Figure 1 | A hippocampal neuronal culture. (a) A single wide-field image of the cells (neurons, green; glia, red). (b) The effects of deblurring the same sample using the built-in cell $\wedge$ R'inverse filter' deconvolution algorithm. For $\mathbf{b}$, a widefield $z$-stack (three-color, 30 image planes, 0.2- $\mathrm{m}$ spacing) was captured using the motorized stage on the Olympus IX81 inverted microscope and a 60X objective. Scale bar, 5 um. Cells were courtesy of L. Allan, M. Bootman and L. Roderick of The Babraham Institute (Cambridge, UK). 


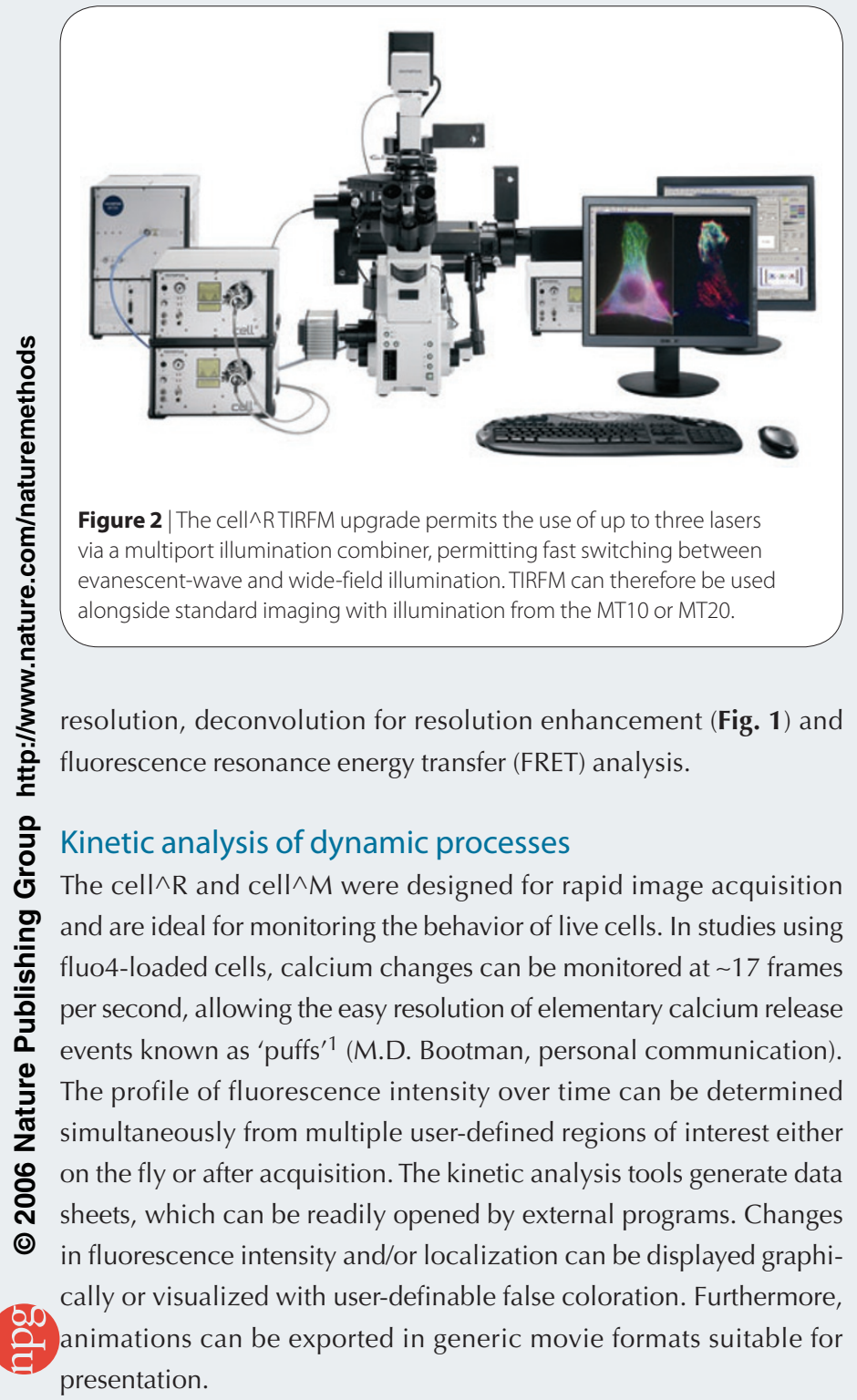

Advanced functionality of the cell $\wedge$ R and cell $\wedge M$

The systems can also incorporate a confocal disk scanning unit (DSU) and a TIRFM module (Fig. 2), both of which are fully integrated with the Experiment Manager.

The Olympus DSU provides a cost-effective solution to obtain confocal sectioning using white-light illumination. The filters used for widefield imaging can also be used for excitation via the DSU. Out-of-focus blur is removed by a spinning disk with slits that generate multiple virtual pinholes when the disk is rotated. Disks with differently spaced slits can be swapped easily by the user to provide the optimum compromise between confocality and light throughput. A single command will rapidly introduce or remove the DSU from the light path, even during an image acquisition series.

Imaging near-membrane processes is problematic using standard confocal technology owing to the small area visible in confocal sections. In studies of protein translocation in living cells, TIRFM has clearly provided a substantially greater sensitivity over confocal
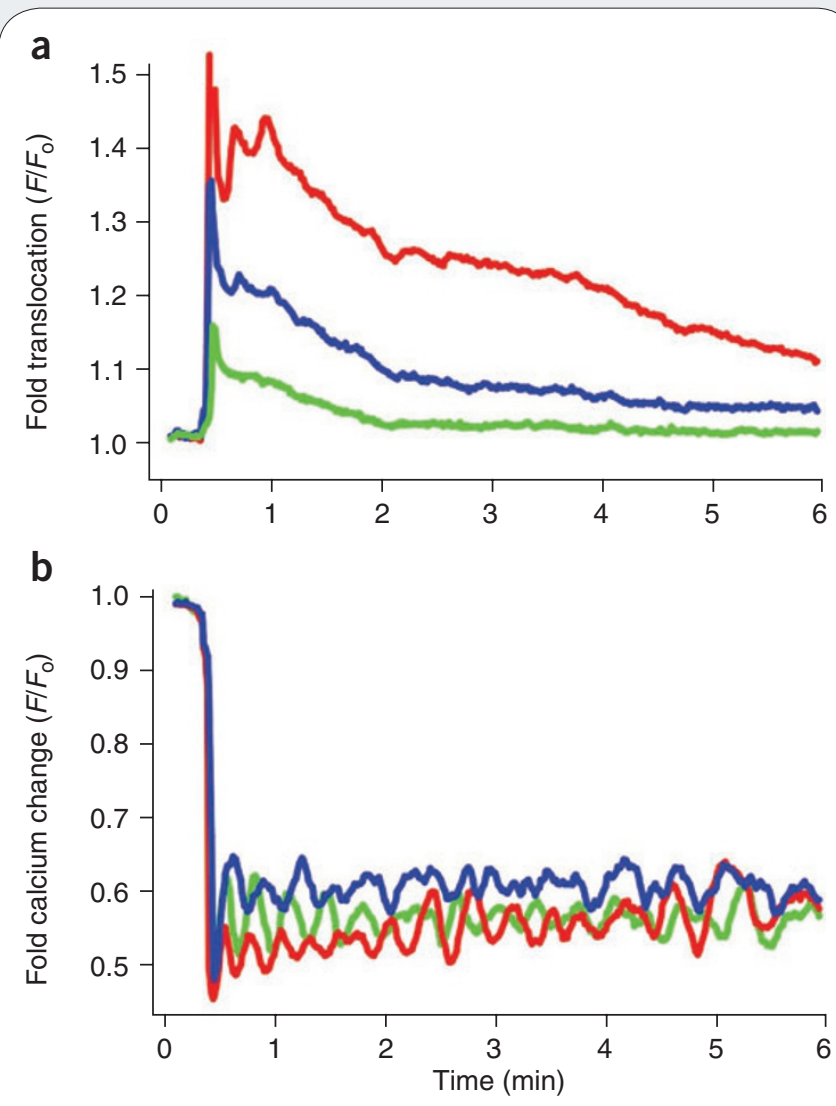

Figure $\mathbf{3} \mid$ Calcium-dependent translocation of a GFP-tagged protein (CAPRI). $(\mathbf{a}, \mathbf{b})$ Fold change in GFP at the basal membrane of three HeLa cells monitored using TIRFM (a) and the concurrent calcium change within the cells (b). The calcium was monitored using wide-field imaging of the fluorescent dye fura2 excited at $380 \mathrm{~nm}$. The downward deflection indicates a rise in intracellular calcium. HeLa cells were stimulated with $100 \mu \mathrm{M}$ histamine for $20 \mathrm{~s}$. The acquisition was switched between TIRFM and wide-field every 0.5 seconds. Data were kindly provided by M. Bootman, S. Walker, L. Roderick and P. Lockyer of The Babraham Institute.

imaging in detecting subtle movements of fluorescently tagged protein ${ }^{2}$. The cell^R TIRFM module permits the use of three separate laser inputs, which can be used sequentially or simultaneously with wide-field illumination (Fig. 3). The laser inputs can be individually adjusted by the user to control the depth of the evanescent waves and are rapidly shuttered $(1 \mathrm{~ms})$. TIRFM places special limitations on objectives, as the light requires large exit angles and therefore large numerical apertures (NAs). Olympus has the largest and most advanced range of TIRFM objectives, from the PLAPON 60xO TIRFM (NA 1.45) to the world leading UAPO 150xO TIRFM (NA 1.45). Moreover, Olympus has produced an APO 100xOHR with a worldleading NA of 1.65 .

1. Bootman, M. D. et al. Cooking with calcium: the recipes for composing global signals from elementary events. Cell 91, 367-373 (1997).

2. Liu, Q. et al. CAPRI and RASAL impose different modes of information processing on Ras due to contrasting temporal filtering of $\mathrm{Ca}^{2+}$. J. Cell Biol. 170 183-190 (2005).

This article was submitted to Nature Methods by a commercial organization and has not been peer reviewed. Nature Methods takes no responsibility for the accuracy or otherwise of the information provided. 